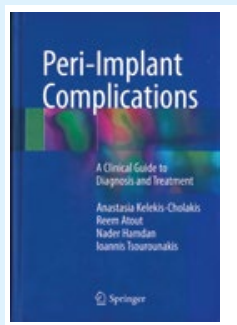

\section{PERI-IMPLANT COMPLICATIONS: A CLINICAL GUIDE TO DIAGNOSIS AND TREATMENT}

Anastasia Kelekis-Cholakis, Reem Atout, Nader Hamdan, Ioannis Tsourounakis

2018; Springer; price f96.50; pp. 117

ISBN: 9783319637174

As the popularity and range of application of dental implants continues to increase, dental clinicians can naturally expect to see a corresponding rise in the number of associated complications in the hard and soft tissues which support them. This book aims to elucidate the peri-implant complications that may occur, and describe their respective aetiologies, diagnoses and treatment options.

Written by a group of distinguished academics and specialists from Canada, this book blends the features of an academic textbook and a narrative review, which is logically laid out and effortless to read. The content of each section is evidence-based, with the relevant citations available at the end of each chapter. Critical appraisal of the relevant literature is provided, and where findings from the literature are equivocal, the authors weigh up the evidence rationally from both sides. The main ideas of each section are summarised in helpfully highlighted boxes at the end of the section, with a concise overall summary to conclude each chapter. The text is well-supported by high-quality images and radiographs showcasing relevant conditions and the steps involved in their diagnosis and treatment.

Despite the book's small size, the authors cover the topic of peri-implant complications in sufficient detail to satisfy the needs of dental practitioners with a wide range of implant experience. For undergraduate students, it would provide a useful entry point for gaining an understanding of the differences between natural teeth and implants, as well as being the 'go-to' source for any queries regarding the tissues that support implants. Equally among general dental practitioners, who may find themselves responsible for the maintenance of more implants than their undergraduate training has prepared them for, this book provides an accessible and usable guide to the diagnosis of peri-implant conditions and treatment options. For the aspiring specialist in periodontology, it will undoubtedly be useful for revising the fundamentals and, above all, is an excellent condensed summary of the available literature in the topic area, with reference material for further reading.

This book can be commended for providing a comprehensive, yet simple, guidance to the oral healthcare team in the long-term monitoring and maintenance of dental implants.

Joey Donovan

\title{
Global Oral Cancer Forum set for Edinburgh 2020
}

A call has gone out across the world to join the assembly of passionate healthcare professionals, policy makers and patients in Edinburgh during March 2020. Following the inaugural Global Oral Cancer Forum (GOCF) held in New York 2016, the second meeting is coming to Scotland, organised and hosted by charity Let's Talk About Mouth Cancer (SC045100).

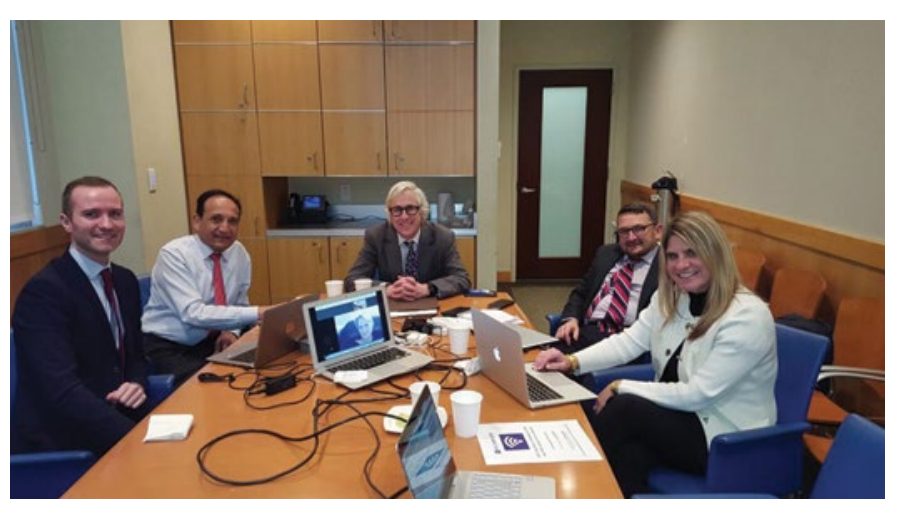

The GOCF'20 planning meeting

Over two days, 6-7 March 2020, the theme for GOCF'20 is 'reducing risk; prevention, early diagnosis and innovative treatments'. A high calibre selection of international speakers, expert panellists and delegates from various world regions will convene to develop actionable outputs in the global battle against oral cancer.

Unlike other international events the GOCF'20 invites delegates from all backgrounds: dentists, doctors, surgeons, nurses, public health practitioners, NGOs, charities, data scientists, survivors and patients to join the conversations and establish new thinking in the challenge oral cancer poses globally.

The innovative programme includes lectures and panel sessions coupled with interactive whole forum conversations such as the World Café on Day 1, where delegates will join with contributors from around the world in a collaborative broadcast session to develop targets and action plans to improve survival and reduce the incidence of new cases of oral cancer. Embracing the future of medicine, the discussion on Day 2 at the 'Big Data, Artificial Intelligence, Precision Medicine forum' aims to inspire new thinking utilising big data and data science to generate novel approaches and solutions.

Speaking as registrations went live, Chair and Executive Director GOCF'20, Professor Victor Lopes said, 'For the GOCF'20 to be a success and initiate real change we need to have delegates from all over the world with different backgrounds and perspectives in attendance. It is really exciting to host the forum here in Edinburgh and the support we've had from local professionals and politicians has been encouraging. We would like to extend the invite out to as wide an audience as possible - come and join the conversation. Only with big ideas and collaboration can we push back and turn the tide on the increasing global burden of oral cancer.

For more information on the GOCF'20 visit: www.gocf20.com or search and follow the social media feeds: Facebook, Twitter and Instagram. \#jointheconversation \#talkinggocf20 